\title{
ALGUNAS APROXIMACIONES AL PROBLEMA DEL CONOCIMIENTO
}

\author{
SOME APPROACHES TO \\ THE PROBLEM OF KNOWLEDGE
}

\author{
Carlos Adolfo Rengifo Castañeda*
}

Rengifo C. Carlos A. Sophia N 7 - 2011. ISSN: 194-8932 Págs. 28- 38.

Recepción: Mayo 30 de 2011

Aceptación: Julio 11 de 2011

\section{RESUMEN}

\begin{abstract}
Este artículo pretende realizar algunas aproximaciones a la epistemología, apoyado de manera primaria y secundaria en autores significativos en torno al devenir de la misma, identificando los elementos tradicionales que constituyeron la propuesta esbozada desde Platón hasta los contra ejemplos presentados por Gettier y, que a su vez, contrastan la noción tripartita presentada por éste filósofo clásico; constituyéndose en una herramienta útil, para efectos de problematizar el conocimiento al interior de la ciencia y fortalecer los procesos de indagación en torno al estatus de cientificidad, objeto de preocupación y carácter de la ciencia en cuestión.
\end{abstract}

PALABRAS CLAVE

Filosofía, Conocimiento, Epistemología, Justificación, Creencia, Normatividad, Racionalidad.

\section{ABSTRACT}

This article is intended to make some approximations to epistemology, primarily and secondarily supported by representative authors towards the incoming of episteme itself, to identify the traditional elements constituting the proposal outlined by Plato before the contra samples presented by Gettier which in turn contrast the tripartite notion stated by this classic philosopher. This constitutesinto a useful tool to the effects of problematizing knowledge at the interior of science and strengthens the process of questioning, towards the status of scientificity being the matter of concern and essence of science itself.

KEY WORDS

Philosophy, Epistemology, Knowledge, Justification, Belief, Normativity, Rationality.

\footnotetext{
Docente de lógica filosófica y epistemología, Universidad La Gran Colombia seccional Armenia. careca1106@gmail.com Co-investigador grupo de investigación PAIDEIA (Categoría A - Colciencias).docenciapedagogia@ugca.edu.co Avenida Bolíbar No. 7-46. Armenia, Quindío, Colombia.
} 


\section{INTRODUCCIÓN}

En la actualidad, en muy diversos círculos académicos o de profesionales, se acude a la epistemología, de manera rápida y despreocupada, quizás por esnobismo, o por el afán de legitimar el discurso de quien se sirve de ésta, o con la pretensión de afincar su argumento sobre una disciplina netamente filosófica; pero podríamos inferir que incurre quien la usa sin beber de la filosofía, en la falacia ad verecundiam, al no ser una autoridad en el tema (teniendo efectos persuasivos, pero no conducentes a la validez del argumento); pero esta afirmación que suena un tanto pretensiosa y arrogante al querer decir que sólo el filósofo puede referirse a la epistemología, también es refutable, en cierto sentido; pues en la contemporaneidad emerge con gran fuerza profesionales de distintas ciencias quienes intentan desde sus recursos cognoscitivos (ya sean sociólogos, psicólogos ${ }^{1}$, biólogos, físicos, químicos, neurocientíficos), indagar por el estatus de cientificidad y/o validez de su ciencia con el rigor académico del caso; reivindicando con esto, el rol de la epistemología al interior del quehacer científico e inclusive generar los distintos giros que se han dado al interior de la epistemología (giro historicista, naturalista entre otros); desde una mirada abierta, pues la "[...] interdisciplinariedad no puede seguir siendo entendida como yuxtaposición de conocimientos o dialogo reduccionista de saberes, esta debe generar nuevos métodos y nuevos objetos pues su desafío es de comprensión en hermenéutica profunda de creación"2, condición que demanda nuevas formas de abordar y problematizar el conocimiento científico.

Por otra parte, en este intento por comprender la ciencia bien sea como actividad humana y/o como su producto, desde una perspectiva epistemológica se hace necesario, indagar en torno al problema de la demarcación ${ }^{3}$ entre lo que es y no es ciencia,

1 Desde la psicología cognitiva se afirma que si el conocimiento es producto de la mente humana, y esta es objeto de preocupación de la psicología, algo respecto al conocimiento puede esta afirmar.

2 Bibiana Vélez Medina, Universidad. Sociedad, Crisis y compromiso. En: Revista Sophia. Ed: No 6, 2010. Pág. 17.

3 "Los intentos por contestar la pregunta ¿qué es la ciencia?, han consistido, desde el Círculo de Viena en adelante, en buscar algún rasgo diferenciador del conocimiento científico que fuera capaz distinguirlo con claridad de otros productos culturales, y en concreto de otras formas pretendidas (ciencia y pseudociencia), lo cual jalona la pregunta por el método; parafraseando a Mario Bunge: donde no hay método no hay ciencia; condición que permite a la ciencia ser objetiva, superando los puntos de vista individuales, y los prejuicios al seguir normas metodológicas.

De ahí que al preguntarse por ejemplo el pedagogo acerca de la pedagogía como ciencia, se vea inmerso en los interrogantes respecto a: ¿Cómo definir el estatus de cientificidad de esta?, ¿Su objeto de preocupación, o quehacer? ¿Cuál es el método(s)? y en últimas ¿Cuál es el horizonte epistemológico, que permite justificar las creencias que desde ella se afirma acerca del mundo?; preguntas esenciales para definir campos de indagación, y/o cuencas de problematización epistémicas; y que en ocasiones son soslayadas por quienes intentan justificar sus saberes, bajo la tutela de la epistemología sin antes haber indagado en torno a tales cuestiones.

Pero en aras a la claridad, se hace necesario conceptualizar en primera instancia acerca de algunos conceptos con los cuales suele confundirse a la epistemología tales como: Filosofía de la Ciencia y la Gnoseología-Teoría del Conocimiento, para la comprensión de los posibles intersticios que permiten definir el estatus de la epistemología en lo que concierne al conocimiento.

\section{ALGUNAS GENERALIDADES ACERCA DEL PROBLEMA}

En un primer momento debemos afirmar que tanto la filosofía de la ciencia, la epistemología y la gnoseología o teoría del conocimiento son discursos o reflexiones de segundo orden, metateorías, estudios metacientíficos; pues teorizan no acerca del mundo, sino en torno a conceptos que tienen como objeto de preocupación la ciencia, el conocimiento científico, siendo la ciencia de primer orden; lo que nos permite inferir un punto de aproximación o de encuentro entre estos discursos.

o no de conocimiento. Es esto lo que se conoce como el problema del criterio de demarcación entre ciencia y no ciencia". Diéguez Lucena Antonio, Filosofía de la Ciencia. Biblioteca Nueva, Universidad de Málaga: Madrid, 2005. Pág. 115 
Por otra parte, la Gnoseología (Nous-vous, גoyoclogos o Teoría del conocimiento, se ha de concebir como el estudio acerca del conocimiento en general, la reflexión en torno a la posibilidad del conocimiento, sin verse en la imperiosa necesidad de analizar aspectos constituyentes del conocimiento como la validez-justificación-racionalidad criterios inherentes a la mirada de la epistemológica y la filosofía de la ciencia. En su lugar, la reflexión filosófica sobre la ciencia (la filosofía de la ciencia) tendrá como objeto "[...] poner de manifiesto o hacer explícitos los aspectos filosófico-conceptuales de la actividad científica, esto es, elucidar conceptos fundamentales de la actividad científica, determinar las normas que rigen esa actividad y reordenar conceptualmente o reconstruir esos sistemas de conceptos producidos por la ciencia que son las teorías"4; pretende comprender las características de la ciencia como conocimiento y los métodos usados por esta a lo largo de la historia; ocupándose de explicar lo que es la ciencia, cómo es posible, sus límites y la naturaleza y evaluación de las teorías científicas.

Ahora bien, la Epistemología ( $\varepsilon \pi \imath \sigma \tau \eta \mu \eta$-ciencia, $\lambda$ oyoc-estudio) pretende entender lo que es conocimiento, pero concretamente conocimiento científico, al intentar indagar por los criterios de validez de la ciencia, desde el punto de vista de su racionalidad-normatividad; se trata de definir el conocimiento alcanzado al interior de una ciencia como racional; cuestiones que se rastrearán a lo largo de este artículo.

Este proyecto epistemológico ha girado en torno a preguntas tales como: ¿Qué es el conocimiento? ¿En qué se fundamenta el conocimiento? ¿Cómo es posible conocer?; las cuales han sido afrontadas a lo largo de la historia de la filosofía desde distintas vertientes. Ferrater Mora ${ }^{5}$ dirá que los griegos introducen en la historia de la tradición filosófica los términos que sirven para expresar dicha cuestión, se trata de los vocablos $\gamma$ voosi "conocimiento" y episthmh "saber", traducido también como "ciencia", sin olvidar que los griegos con frecuencia trataron problemas gnoseológicos, pero subordinándolos a cuestiones luego llamadas "ontológicas".

4 Moulines Ulises, Díez José A. Fundamentos de filosofía de la ciencia. Editorial: Ariel, Barcelona, Pág. 25

5 Ferrater Mora, José. Diccionario Filosófico. Ed: Sudamericana, Buenos Aires. Pág. 329
Es así como a lo largo de la historia la pregunta "¿Qué es el conocimiento?" es ideada por los griegos con una fuerte imbricación a la pregunta "¿Qué es la realidad?", asunto problematizado y comprendido de manera similar en algunos filósofos medievales.

Sin embargo, según Ferrrater Mora, "[...] es plausible sostener que sólo en la época Moderna (con varios autores renacentistas interesados por el método y con Descartes, Malebranche, Leibniz, Locke, Berkeley, Hume y otros) el problema del conocimiento se convierte a menudo en problema. Pero todavía no se concebía el estudio del conocimiento como pueden dar impulso a una -disciplina filosófica especial-. Desde Kant, en cambio, el problema del conocimiento comenzó a ser objeto de la teoría del conocimiento. La filosofía de Kant no puede reducirse, como lo han pretendido algunos neokantianos, a teoría del conocimiento. Pero es indudable que esta teoría ocupa un lugar muy destacado en el pensamiento de dicho filósofo. Desde Kant, además, se ha manifestado con frecuencia en el pensamiento filosófico moderno y contemporáneo una cierta "epistemofilia", que contrasta con la "ontofilia" de los griegos y de muchos autores medievales"6.

De este modo, se percibe cómo ésta tradición epistemológica alcanza su madurez programática en Kant quien, según Rodríguez Alcázar, le atribuye a la hasta entonces teoría del conocimiento "[...] la potestad de otorgar, desde la posición privilegiada de un Tribunal Supremo, el marchamo de legitimidad científica, al tiempo que desacreditó de forma casi irreversible la previa concepción del saber que hacía de la metafísica la Reina de las ciencias; esto último significa, en primer lugar, que a partir de Kant la epistemología ocupará la posición privilegiada que antes correspondía a la metafísica"7, transformando a su paso las formas de relación entre la ciencia y la filosofía, haciendo de la epistemología una disciplina normativa de orden superior a las demás disciplinas científicas, y demarcando a la ciencia con respecto a la filosofía, (demarcación que será objeto de indagación

\footnotetext{
Ídem.

7 Rodríguez Alcázar, Francisco Javier. "la radicalización del naturalismo" En: Revista de Filosofía, $3^{\underline{a}}$ época, vol. VIII (1995), núm. 14, Universidad Complutense de Madrid. Pág. 109
} 
a la luz de algunos representantes del programa naturalista).

Sin embargo, el asunto epistemológico se ha centrado en tres aspectos fundamentales:

(1) Definir el conocimiento.

(2) Enfrentar los cuestionamientos planteados por el escéptico, quien pretende mediante un constante preguntar, constreñir a quien afirma algún tipo de saber. Preguntas que en la mayoría de los casos incurren en la falacia de petitio principii.

(3) Analizar los aspectos constitutivos en la producción de creencias, determinando a su vez la relación de estos con el conocimiento.

El aspecto tres ha sido uno de los pilares de desarrollo de la epistemología y desde el cual, en la contemporaneidad (giro naturalista), se realiza un cuestionamiento importante respecto a la manera como tradicionalmente se ha entendido las creencias, su justificación y el carácter normativo de la epistemología.

De lo anterior se sigue que abordar la cuestión acerca del conocimiento desde la Epistemología, implique cuestionar el problema acerca de la naturaleza de éste y el de la justificación de las creencias, problematizando la clásica caracterización respecto al conocimiento en la que éste se comprende como una creencia, verdadera y justificada; concepción que ha variado sus interpretaciones a lo largo de la historia, pero que en esencia conserva los pilares constitutivos del mismo.

\section{La Noción Tradicional de Conocimiento}

Desde la llamada definición clásica o tradicional se pensaba que el conocimiento era el resultado de una sumatoria de productos, tal ecuación compuesta por tres partes (tripartita) afirma que este es el efecto de las sucesivas condiciones: creencia + justificación + verdad = conocimiento.

De forma tal que la creencia verdadera está adecuadamente justificada 0 , si se quiere, que el enunciado $S$ sabe (o conoce) que $P$ tiene tres condiciones constitutivas que de manera ilustrada pueden ser analizadas a partir de lo siguiente:
$S$ sabe o conoce a $P$ si y sólo sí

1. $P$ es verdadera

2. $S$ cree que $P$

3. $S$ está justificado a creer que $P$

Siendo $P$ el contenido proposicional del cual se afirma (si es verdad), es decir, algún tipo de conocimiento, $S$ será el sujeto epistémico o cognoscente, quien deposita su conocimiento respecto a $P$, y que además ha de tener las razones, la justificación del mismo.

1. P es verdadera: Juan es meteorólogo, y al analizar las distintas variaciones climáticas en los últimos días concluye que habrá un tornado de grandes magnitudes, es decir, Juan cree la verdad de tal fenómeno.

2. $S$ cree que $P$ : Juan apoyado en su formación académica y en los instrumentos propios de su oficio cree adecuadamente la verdad respecto al tornado y.

3. S está justificado a creer que $P$ : Efectivamente es verdad y está justificado para Juan, que tendrá lugar el tornado, esto a razón de las evidencias proporcionadas por su formación e instrumentos de su quehacer como meteorólogo.

En consecuencia de esto las condiciones constitutivas de la definición tripartita, permiten inferir que Juan tiene conocimiento, dando respuesta a su vez a los cuestionamientos acerca de si Juan sabe lo que cree saber, ¿de qué manera lo sabe?, ¿cómo llegó a saber lo que sabe? y si efectivamente está justificado.

Frente a tales argumentos el escéptico replica:

"[...] 1. Nadie sabe que $p$ a no ser que pueda decir cómo sabe que p. 2. No es posible contestar satisfactoriamente a la cuestión ¿Cómo sabes que $p$ ? por el mero procedimiento de volver a aseverar que $p$. Se trata de una petición de principio"8, dejando clara su intención de atacar directamente la noción del conocimiento y/o la noción de justificación de este; por lo cual, sería imposible afirmar tal conocimiento por parte de

8 Dancy, Jonathan. Introducción a la epistemología contemporánea. Ed: Tecnos. Madrid. 1993. pág. 21 
Juan, pues los argumentos a favor de justificar que efectivamente se da tal tornado son imposibles; agregando el escéptico que nadie puede saber nada; y para el caso de Juan acerca de su predicción, de su creencia.

En 1963 Edmund Gettier mediante una serie de contraejemplos afirmó que no bastan las tres condiciones clásicas expuestas por Platón para decir que conocimiento es el expresado por un sujeto $S$ quien sabe que la proposición $p$ es verdadera; y planteó, de inmediato, la de agregar la condición o las condiciones para dar cuenta de que un sujeto $S$ acepte una proposición $p$-evidente y verdadera- pero conociendo que $p$ es verdadera.

Uno de los contraejemplos citados por Jonathan Dancy, con los cuales Gettier analiza la noción tripartita del conocimiento y argumenta que ésta es insuficiente, aún si lo que se cree saber cumple con las tres clausulas, es el siguiente:

“[...] Henry está mirando la televisión una tarde de junio. Es la final masculina del torneo de Wimbledon y McEnroe está venciendo a Connors por dos sets a uno y con punto de partido en el tercero. McEnroe gana este punto. Henry cree justificadamente que:

1. Acabo de ver la victoria de McEnroe en la final de Wimbledon de este año

E infiere razonablemente que

2. McEnroe es el campeón de Wimbledon de este año

Sin embargo, lo que ha sucedido es que las cámaras instaladas en Wimbledon se han estropeado y lo que muestra la pantalla es una grabación del partido del año anterior. Mientras sucede eso, McEnroe está, de hecho, a punto de repetir la abultada victoria que obtuvo el año pasado. De modo que la creencia 2 de Henry es verdadera, y está ciertamente justificado para tener tal creencia. Pero no concederíamos que Henry sabe 2" 9 .

Pensemos del mismo modo en otro contraejemplo pero desde otra perspectiva. El caso de la resurrección de los muertos en la tradición cristiana.

9 Ibíd. Pág. 40
El apóstol Pedro afirma su firme creencia en ella; pues Jesús su maestro le había trasmitido tal creencia. Pedro como apóstol de la iglesia trasmite tal contenido proposicional como verdadero. Lo mismo hace el apóstol Pablo quien sin conocer a Jesús se dirige a un ágora bastante exigente como la ateniense (recordemos que el Areópago había juzgado y condenado a muerte a Sócrates). Pablo según lo narra el texto bíblico, Los Hechos de los Apóstoles, inicia su discurso hablando de un "tal" Jesús que a oídos de los atenienses resulta ser interesante hasta que habla de la resurrección de los muertos, creencia rechazada por los habitantes de Atenas, quienes comienzan a abandonar el areópago; para un griego no era creíble tales argumentos; y expresaron cortésmente al orador: Pablo, te escucharemos con gusto otra vez, dejándolo sólo.

Pablo no encuentra nuevas premisas que justifiquen su creencia sobre la resurrección y agrega: "estos griegos atenienses buscan sólo sabiduría, y yo no enseño más sabiduría que la de la Cruz".

De lo anterior se puede inferir que Pablo no tenía conocimiento, pues sin justificación no hay tal (contrastando con esto, la noción tripartita expuesta por Platón: creencia-verdad-justificación). Pablo tiene una proposición que al interior de la primera comunidad cristiana es verdadera y justificada; sin embargo, las preguntas y cuestionamientos esgrimidos por los atenienses (desde una postura escéptica) lo conducen a esfuerzos infructuosos.

Tal argumento en torno a la resurrección demanda de nuevas pruebas (premisas) que la justifiquen; de ahí la importancia para la teología cristiana del sepulcro vacío, prueba ontológica de la resurrección de Jesús y que permite afirmar "si no hay cuerpo es porque Jesús ha resucitado"; por consiguiente, la creencia en la resurrección a la luz de esta nueva premisa puede confirmar la proposición de Pablo, como conocimiento; reviviendo si se quiere la discusión acerca de tal creencia.

En efecto, se debe decir, que aun teniendo una proposición y la creencia en ella como verdadera, pero no su justificación no se puede inferir de ello que se tiene conocimiento, pues sin justificación no hay conocimiento; siendo así que cada una de tales condiciones son necesarias por sí solas, 
pero no suficientes. Gettier no pone en tela de juicio el carácter de necesarias de las tres clausulas, pero sí argumenta que no son suficientes, de modo tal que: "[...] en un contraejemplo de Gettier, a tiene una creencia justificada pero falsa, de la cual infiere la creencia justificada en algo que de hecho es verdad, llegando así a una creencia justificada y verdadera que no es conocimiento"10. De lo anterior se sigue, que puede haber creencia sin justificación, y que por tanto, no satisfacen la definición tripartita del conocimiento. Según Gettier, a partir de sus contraejemplos, se demuestra que la definición de conocimiento como creencia verdadera justificada es errónea, siendo imperioso un abordaje conceptual distinto acerca del conocimiento.

En este contexto y a manera de comentario, pues no será objeto de análisis detallado en este escrito, podemos recordar que en 1967 Alvin Goldman "[...] agrega una cuarta condición y es la de que la proposición $p$ esté causalmente conectada de manera -apropiada- con la creencia de $S$ de que $p^{\prime \prime 11}$, lo que se conoce como teoría causal del conocimiento. Para este fin, Goldman propone tomar en cuenta los procesos causales que hacen que una persona crea algo y cómo este proceso contribuye en la configuración y justificación de la creencia; reconociendo con esto que los procesos causales confieren justificación a las creencias y tienen la condición de hacerla confiable. De este modo, Goldman proyecta su propuesta en torno a la justificación de una creencia con base en la confiabilidad del proceso o los procesos que la causan. Esta confiabilidad consiste en la tendencia de un proceso a producir creencias que sean verdaderas en lugar que falsas.

Conforme a lo anterior, el vínculo referido a las tres condiciones constitutivas y esenciales de la definición clásica del conocimiento, ha sido tradicionalmente considerado como necesario, pero no suficiente al intentar precisar la condición (2) acerca de si " $S$ tiene una justificación adecuada en favor de la verdad de $P$ ".

Estas condiciones serán el hilo conductor de este

10 lbíd. Pág. 42

11 Filosofía de las ciencias naturales, sociales y matemáticas. Ed. Anna Estanny. Madrid: Trotta, 2005, Pág. 31 problema respecto a las acciones epistémicas, necesarias y suficientes, para fundamentar el conocimiento, es decir, a la hora de obtener las justificaciones suficientes acerca de las afirmaciones que se presentan respecto del conocimiento y que para la contemporaneidad se convierte en caballo de batalla a la luz de algunos giros epistemológicos (giro historicista, naturalista).

No obstante, con esto se intenta recordar, que la reflexión epistemológica tradicional tiene como uno de sus propósitos más sentidos abordar la noción de creer que, estar justificado a, y entre otras proporcionar la fundamentación última del conocimiento, tratando a su paso de dar respuesta a las objeciones presentadas por el escéptico, quien pone en tela de juicio la plena comprensión y alcance de la realidad por parte de los sujetos, al afirmar que nadie puede saber nada de hecho; por esto la importancia de recordar que uno de los temas dominantes del escepticismo desde la antigüedad, según Francisco Javier Rodríguez Alcázar es la observación de que toda argumentación es objetable porque:

"[...] en último término, cae inevitablemente en una regresión infinita o en un razonamiento circular. La tradición epistemológica moderna acepta generalmente el campo de batalla propuesto por el escéptico, comprometiéndose en el intento por escapar a esos dos vicios. De ahí que esa tradición haya asumido que la justificación del conocimiento debe cumplir dos condiciones para ser convincente. En primer lugar, dicha justificación habría de incluir la identificación de alguna clase de creencias privilegiadas que se pudieran considerar fuera de toda duda y que, por eso mismo, sirvieran como fundamento último al resto del conocimiento; de este modo se evitaría la regresión infinita. En segundo lugar, evitar la caída en la circularidad hace necesario que la justificación del conocimiento se lleve a cabo sin hacer uso de los resultados ofrecidos por la misma ciencia que se pretende legitimar; la epistemología habrá de emplear, pues, una metodología a priori que, unida a su talante normativo, la separa de las ciencias. Aceptadas estas dos condiciones, la epistemología queda instituida como una filosofía primera que proporciona, desde fuera, el fundamento que parecen necesitar las ciencias"12.

En consecuencia, la epistemología tradicional se

12 Rodríguez Alcázar, Francisco Javier. "la radicalización del naturalismo" Pág. 110. 
encuentra inmersa en la discusión acerca de la justificación de las creencias y fundamentación del conocimiento, discusión que no será ajena a otras propuestas epistemológicas como la naturalización de la epistemología o el giro naturalista en epistemología, en la que la epistemología se adentra en las cuestiones acerca de las creencia y la justificación, sin temor alguno a caer en un regresum ad infinitum que lo conduzca a una circularidad viciosa al hacer uso de los resultados dados por la misma ciencia que se pretende legitimar; sin por esto, abandonar su carácter de normativa.

\section{La noción de creencia}

De manera cotidiana se afirma que la creencia, como uno de los componentes del conocimiento, es todo aquello respecto a lo cual el sujeto afirma conocer o saber algo, la cual emerge cuando se acepta que una proposición es verdadera; "[...] las creencias cognitivas están desde esta perspectiva epistemológica, radicalmente vinculadas a las proposiciones. Desde la filosofía de la mente las creencias son estados mentales verdaderos o falsos que tienen como contenido proposiciones, así el contenido representacional de las creencias son las proposiciones, siendo estas una parte constitutiva de las creencias"13.

Tener una creencia como componente epistémico acerca del mundo no implica necesariamente, como se ha dicho, que tal proposición que se emita sea verdadera. Afirmar una creencia implica aceptar que no se cuenta con plena seguridad respecto del contenido de la misma; lo cual demanda razones o evidencias que la soporten. Tal situación concerniente a las creencias serán abordadas desde cuestiones más técnicas de la filosofía, como son el racionalismo y empirismo, perspectivas que problematizan las creencias bien sea por vía de la razón, o por vía de la experiencia, y que recuerda la típica discusión histórica dada entre racionalistas y empiristas, en la cual René Descartes, por un lado, y John Locke, por el otro, pensaban que habían hallado el proceso de justificación de las creencias a partir de un fundamento seguro.

Descartes explica las creencias a partir de las

13 Posada R. Gregorio. La definición tripartita del conocimiento, un análisis desde el lenguaje ordinario. En: Revista de Ciencias Humanas, UTP, No. 35 Enero- Junio de 2005. Pág. 49 ideas claras y distintas, es decir, en lo manifiesto desde ellas; Locke, por su parte, las explica a la luz de las ideas simples que provenían de nuestras percepciones. Al respecto y de manera un tanto somera, se intentará rastrear la noción de creencia en la epistemología tradicional, en aras de comprender tal componente esencial del conocimiento. Este asunto acerca de las teorías de las creencias, se verá fuertemente imbricado con la teoría de la justificación, la cual pretende esclarecer o, mejor, sugerir desde qué aspectos una creencia puede aceptarse como verdadera o falsa.

Para efectos de comprensión de las condiciones incidentes en la reflexión epistemológica se realizará una breve aproximación a lo que respecto de las creencias dirán Locke, Hume, Peirce y Descartes. John Locke (1632-1704), pensador inglés, es considerado uno de los máximos representantes de la doctrina filosófica del empirismo, a través de su obra Ensayo sobre el entendimiento humano (1690). David Hume (1711-1776), filósofo, historiador y economista escocés, quien ejerció una notable influencia en el desarrollo del escepticismo y del empirismo, en su Tratado sobre la naturaleza humana (1739) expone las principales ideas de su pensamiento filosófico y presenta a las creencias como un sentimiento vivaz que acompaña a las ideas, en las que se depositan posteriormente las creencias. Para finalmente abordar a Peirce (1839-1914), filósofo y físico estadounidense que sentó las bases de la semiótica. Omitiendo intencionalmente en ésta sucinta presentación, como es claro, lo que al respecto dicen ilustres exponentes del racionalismo, como Spinoza y Leibniz, pues la pretensión aquí no es revivir la discusión entre la escuela británica y continental, sino realizar una breve aproximación al asunto relativo a las creencias, sin embargo, de manera un tanto rápida se abordarán algunos aspectos relevantes en la propuesta epistemológica desarrollada por Rene Descartes, con la pretensión de mostrar el contraste con el empirismo y cómo ambas perspectivas, empirismo y racionalismo, son fundacionalistas.

John Locke plantea los fundamentos del conocimiento humano, apoyado en la experiencia. Por tal razón, resulta importante comenzar recordando que para los empiristas la mente era vista como un papel en blanco, Nihil in mente quod non prius 
in sensu, agregando:

"[...] Supongamos, pues, que la mente sea, como se dice, un papel en blanco, limpio de toda instrucción, sin ninguna idea. ¿Cómo llega entonces a tenerla? ¿De dónde se hace la mente con esa prodigiosa cantidad que la imaginación limitada y activa del hombre ha grabado en ella, con una variedad casi infinita? ¿De dónde extrae todo ese material de la razón y del conocimiento? A estas preguntas contesto con una sola palabra de la experiencia, he aquí todo el fundamento de nuestro saber, y de donde en última instancia se deriva: Las observaciones que hacemos sobre los objetos sensibles externos, o sobre las operaciones internas de nuestra mente, las cuales percibimos, y sobre las que reflexionamos nosotros mismos, son lo que proveen a nuestro entendimiento de todos los materiales del pensar. Estas son las dos fuentes de conocimiento de donde parten todas las ideas que tenemos o podemos tener de manera natural"14.

Por su parte, Hume abordará este problema valiéndose, como buen empirista moderno, de la información que proporcionan los sentidos al entendimiento humano, sin embargo, advierte "[...] sobre las dificultades de una completa teoría de la creencia"15, pues, aun cuando cree entender perfectamente el tema, afirma no encontrar los términos para expresar lo que desea. Cuatro son las nociones fundamentales que se han de tener presentes a la hora de intentar comprender la explicación elaborada por Hume sobre las creencias: la facultad de la imaginación, las ideas, las impresiones y la vivacidad. En el entendimiento, "[...] la facultad de la imaginación es la encargada de configurar o representar el cúmulo de datos sensoriales en las imágenes o ideas de los objetos. Es gracias a la imaginación que los datos de los sentidos se ordenan y se muestran como ideas que representan el mundo"16.

Siendo claro que tales facultades pueden copiar

14 Locke, John. Ensayo sobre el entendimiento humano. Ed: 1920. Pág. 164

15 Posada Ramírez, Jorge Gregorio. La Noción tripartita del conocimiento: Una introducción a la epistemología. Ed: Cuadernos filosóficos literarios. Universidad de Caldas. 2007. Pág. 40 (Para éste momento acerca de las creencias resulta de gran importancia lo expuesto por el profesor Posada con quien he adelantado varios diálogos al respecto los cuales han sido de gran utilidad).

16 Ibid. o imitar las impresiones proporcionadas por los sentidos, pero nunca alcanzar la fuerza o vivacidad de la experiencia misma. Es así como Hume, en su intento por explicar todo el material constitutivo del pensar humano, se ve en la necesidad de distinguir las percepciones de la mente en dos clases o especies, que en palabras suyas, se diferenciarán por sus distintos grados de fuerza o vivacidad. Una clase de percepción será llamada de pensamientos o ideas, caracterizada por ser las menos fuertes e intensas y la otra será llamada percepción de impresiones, con las cuales pretende denotar aquellas percepciones más intensas, tales como las suministradas por los sentidos: el oír, el ver, el sentir, el amar, el odiar, el desear.

La percepción de ideas, será vista como la copia de la percepción de impresiones, las cuales son comprendidas a partir de su teoría de la asociación de ideas, permitiendo de este modo esclarecer la causa de la cualidad de vivacidad que acompaña a algunas ideas y posibilita la creencia en ella; pues "[...] las ideas presentes en la memoria y en la imaginación no discurren al arbitrio, están conectadas por principios de asociación que garantizan el discurrir normal y coherente de nuestros pensamientos"17, proponiendo así, tres principios de interconexión de las ideas "[...] semejanza, contigüidad y causa y efecto. (Hume) Justifica estos tres principios basándose en acontecimientos mentales corrientes. Por semejanza, la sensación de un retrato nos lleva a la idea del original; por contigüidad, cuando nos acercamos a la casa del vecino aparece en la mente la idea de nuestra casa; por causa y efecto, cuando recibimos el correo del ser extrañado aparecen en nuestra mente ideas sobre él"18, principios de asociación de ideas que no sólo interconectan ideas entre sí, sino que también traen consigo la vivacidad de las impresiones; quedando así, insinuada la concepción de Hume, acerca de la creencia: la vivacidad de las ideas y su relación o asociación con la percepción de impresiones.

Siguiendo con este asunto relativo a la creencia empirista, se encuentra Peirce, quien considera que el conocimiento tiene su fuente en la búsqueda de creencias estables, siendo estas la meta a la cual tiende el razonamiento, la indagación o

17 Ibíd. Pág. 46

18 Ibid. 
investigación, a partir de inferencias de la realidad. Peirce diferencia cuatro métodos para el afianzamiento de las creencias de la siguiente manera:

El primer método, "[...] el denominado método de la tenacidad, estriba en adoptar una opinión y sostenerla obstinadamente sin atender a nada de lo que pueda contradecirla siguiendo la táctica de la avestruz... El segundo el de la autoridad, consigue esto a primera vista. Mediante la violencia y la coacción es posible mantener un rígido control sobre las doctrinas en un país determinado... Otro medio de afianzar la creencia, el método apriorístico, que es evidentemente mucho más intelectual y respetable desde el punto de vista de la razón. La fuerza de este nuevo procedimiento descansa justamente en que no se atiene a la preferencia ni al caprichoso mandato del individuo o del grupo social, sino a lo que parece conformarse con los dictados de la razón. Pero su condición metafísica, el hecho de no ajustarse a la experiencia, sino a las inclinaciones de lo que se reputa como perteneciente a la naturaleza humana, constituye asimismo su mayor debilidad. El cuarto método, el único capaz de satisfacer nuestras dudas, es el método científico. En él nuestras creencias no son determinadas por nada humano, sino por algo externo y permanente, sobre lo cual, nuestro pensamiento no ejerce ningún efecto. Su hipótesis fundamental es que existen cosas reales, cuyos caracteres son enteramente independientes de nuestras opiniones sobre ellos; estas realidades afectan nuestros sentidos según leyes regulares, de conformidad con las cuales y mediante el razonamiento podemos averiguar cómo son las cosas real y verdaderamente"19

Por otra parte, desde la orilla racionalista se encuentra la obra del filósofo francés René Descartes (1596-1650) considerada pilar fundamental en la filosofía moderna. Con ella se materializan algunos conceptos que juegan un papel importante en el desarrollo del pensamiento moderno y en lo concerniente a la construcción del conocimiento científico. René Descartes, a partir de su Discurso del Método (1637 editado en francés), ofrece una exposición de los procedimientos lógicos y científicos que subyacen a su método racionalista que tiene como atributo ascender de

19 Peirce, S. Charles. Deducción, Inducción e Hipótesis. Ed: Aguilar, Argentina. 1970. Pág. 17-19 forma gradual de lo más fácil y simple de conocer, hasta los más complejos. La duda se constituye en el primer momento del método; apoyado en ésta derriba todos los conocimientos alcanzados "[...] no sólo los de los libros, sino, en general, todas las opiniones recibidas. El inservible edificio está derribado" 20 , quedando sólo el mundo y el yo, agregando Descartes que en lo que atañe a las opiniones que había admitido en su creencia, "[...] no podía hacer cosa mejor que intentar por una vez suprimirlas todas, a fin de colocar después en su lugar, bien otras mejores o bien las mismas, una vez ajustadas al nivel de la razón"21, abordando a su paso, los problemas de Dios, del hombre y del alma desde una perspectiva filosófica y científica; para tal efecto entonces, era necesario:

"[...] Primero. No aceptar nunca cosa alguna como verdadera que no la conociese evidentemente como tal, es decir, evitar cuidadosamente la precipitación y la prevención y no admitir en mis juicios nada más que lo que se presentase a mi espíritu tan clara y distintamente, que no tuviese ocasión alguna de ponerlo en duda. El segundo: dividir cada una de las dificultades que examinase en tantas partes como fuera posible y como se requiriese para su mejor resolución. Tercero: conducir ordenadamente mis pensamientos, comenzando por los objetos más simples y fáciles de conocer para ascender poco a poco como por grados, hasta el conocimiento de los más complejos, suponiendo, incluso, un orden entre los que no se preceden naturalmente. $\mathrm{Y}$ el último: hacer en todas partes enumeraciones tan completas y revistas tan generales que estuviese seguro de no omitir nada"22.

Sin embargo, tal método es juzgado por el mismo Descartes como insuficiente, pues era este "[...] sólo una parte de su método racionalista. [...] El hombre nuevo en Descartes -el hombre dirigido por su método- encuentra en las Meditaciones metafísicas un complemento formativo más sereno, e incluso una lógica más segura y de más aplicación en todos los campos de la ciencia"23; apartando la mente de los sentidos y de los prejuicios que estos fundan, lo que a su vez conduce a refutar las creencias justificadas

20 Descartes, Rene. El Discurso del Método. Ed: Aguilar. 1972. Pág. 13-14

21 Ibíd. Pág. 50

22 Descartes, Rene. El Discurso del Método. Pág. 55-56 
a la base de la ilusión sensorial. El testimonio brindado por los sentidos en el proceso de construcción de las creencias será a partir de aquí considerado engañoso; de ahí la importancia de la duda, como condición necesaria para caminar hacia la certeza, hacía el conocimiento claro y distinto. De esta manera, la duda metódica permite tamizar las creencias a la base de las cuales se construye el edificio del conocimiento; para tal efecto, "[...] el argumento de la ilusión sensorial, el argumento del sueño y el argumento del genio maligno son las pruebas a las que progresivamente son sometidas las creencias para determinar cuáles son dubitables, y, en consecuencia, tratadas como si fuesen falsas, y cuales son claras, evidentes y distintas" ${ }^{24}$; en razón de esto, las creencias podrán ser justificadas y asumidas como firmes

Todo lo hasta aquí expuesto indica cómo la creencia racionalista de que el conocimiento tiene su origen en la intuición, o en el innatismo de las ideas expuesto por Descartes, y que a su vez contrasta con la postura empirista de la percepción proporcionada por los sentidos a la mente, como la principal prueba y fuente de toda creencia, todo conocimiento. En consecuencia, en términos generales, se define una creencia como la aceptación por parte del sujeto de algo como verdadero. Es así como, las creencias ocupan"[...] el lugar del componente psicológico subjetivo, a partir del cual se construye el estado de conocimiento, con el cual se pretende alcanzar verdades que tienen una validez objetiva. Otras veces, se considera que el estado de creencia es un estado mental distinto e inferior al del conocimiento y que se tiene en su defecto"25.

Ahora bien, como se ha venido esgrimiendo hasta el momento, las creencias se han de entender en relación con el intento por justificarlas, de ahí que la teoría de la justificación fundacionista se ocupe "[...] de analizar la condición tres de la definición tripartita del conocimiento: S está justificado a creer que $p^{\prime 26}$, intentando comprender los interrogantes

23 Descartes, Rene. Meditaciones Metafísicas. Ed: Aguilar. Argentina. 1970. Pág. 15

24 Posada Ramírez, Jorge Gregorio. La Noción tripartita del conocimiento: Una introducción a la epistemología. Pág. 106

25 Villoro, Luis. El conocimiento, Enciclopedia iberoamericana de filosofía, № 20, Trotta y CSIC, Madrid 1999, p. 63. acerca de ¿Qué hace que una creencia sea justificada?; el fundacionalismo trata de abordar tal inquietud al considerar que las creencias se dividen en dos grupos: "[...] las que necesitan el apoyo de otras y las que pueden apoyar a otras, sin necesitar ellas mismas ningún tipo de fundamentación. Estas últimas constituyen nuestros fundamentos epistemológicos; los primeros, la superestructura construida sobre esos fundamentos" 27 .

En lo referente a las creencias básicas, el fundacionalismo agrega que estas: "[...] son creencias relativas a la naturaleza de nuestros propios estados sensoriales, o nuestra experiencia inmediata. Tales creencias descansan en sus propios pies, sin apoyarse en otros"28. En otras palabras, para el fundacionismo clásico nuestro conocimiento deriva de las experiencias; siendo tales creencias infalibles, al estar fundadas en los datos de la experiencia. A raíz de lo anterior, se comprende a la epistemología según el fundamentalismo como "[...]un programa de investigación que trata de mostrar cómo es posible que nuestras creencias sobre el mundo externo, sobre la ciencia, sobre el pasado y el futuro, sobre las otras mentes etc.. puedan justificarse sobre una base que está restringida a las creencias infalibles sobre nuestros propios estados sensoriales"29; situación que será contrastada y refutada en la contemporaneidad a la luz del programa naturalista o de naturalización y que ha conducido a la epistemología tradicional a una situación de crisis.

\section{RESULTADOS}

Este artículo presenta desde una perspectiva analítica, resultados preliminares de una investigación en torno a la epistemología tradicional e insinúa la crisis que para la contemporaneidad circundará a la epistemología tradicional a la luz del giro naturalista, giro que se vislumbra en este escrito, pero no se desarrolla suficientemente. Por otra, apoyados en esta mirada aproximada del problema, nos vemos conducidos por el mundo de la filosofía,

26 Posada Ramírez, La Noción tripartita del conocimiento: Una introducción a la epistemología.Pág. 78

27 Dancy, Jonathan. Introducción a la epistemología contemporánea. Pág. 71

28 Ídem.

29 Posada Ramírez, La Noción tripartita del conocimiento: Una introducción a la epistemología.Pág. 80. 
por el mundo de la epistemología, para en este viaje luego de interactuar con la epistemología tradicional renunciar a todos aquellos criterios apriorísticos y metametodológicos que confinaban desde ella, la práctica científica a una misma realidad, a un mismo origen, a una única forma de ver el conocimiento; esto es, a reconocer cómo el conocimiento no es sólo una creencia-verdaderajustificada, noción tripartita, como ya lo había corroborado Gettier, pues además del sujeto epistémico existe un flujo torrencial, natural y contextual de información al cual pertenece de algún modo el conocimiento, llegándose a contrastar y refutar la noción de justificación del conocimiento acuñada por la epistemología tradicional: las creencias, y el modo de justificarlas entran en cuarentena y con ellas los intentos fundacionalista a prioristas, a raíz del surgimiento del naturalismo, cuya máxima es "no hay tal filosofía primera" (argumentos que quedan en punta en esta parte de la investigación).

Por último, se espera que éste artículo sirva no sólo para el docente de epistemología, sino también para todo aquel que con un interés en particular desea adentrarse en estas vicisitudes filosóficas, siendo así este un instrumento de problematización e indagación, para efectos de comprensión de la epistemología y del estatus de cientificidad de quien a la luz de su horizonte conceptual desea comprender las ciencias.

\section{REFERENCIAS BIBLIOGRÁFICAS}

Bibiana Vélez Medina, Universidad. Sociedad, Crisis y compromiso. En: Revista Sophia. Ed: No 6, 2010

Dancy, Jonathan. Introducción a la epistemología contemporánea. Ed: Tecnos. Madrid. 1993.

Descartes, Rene. El Discurso del Método. Ed: Aguilar. 1972

Descartes, Rene. Meditaciones Metafísicas. Ed: Aguilar. Argentina. 1970.

Diéguez Lucena Antonio, Filosofía de la Ciencia. Biblioteca Nueva, Universidad de Málaga: Madrid, 2005.

Ferrater Mora, José. Diccionario Filosófico. Ed: Sudamericana, Buenos Aires.

Filosofía de las ciencias naturales, sociales y matemáticas. Ed. Anna Estanny. Madrid: Trotta, 2005.

Locke, John. Ensayo sobre el entendimiento humano. Ed: 1920 Moulines Ulises, Díez José A. Fundamentos de filosofía de la ciencia. Editorial: Ariel, Barcelona.
Posada R. Gregorio. La definición tripartita del conocimiento, un análisis desde el lenguaje ordinario. En: Revista de Ciencias Humanas, UTP, No. 35 Enero - Junio de 2005.

Peirce, S. Charles. Deducción, Inducción e Hipótesis. Ed: Aguilar, Argentina. 1970.

Rodríguez Alcázar, Francisco Javier. "la radicalización del naturalismo" En: Revista de Filosofía, 3를 época, vol. VIII (1995), núm. 14, Universidad Complutense de Madrid.

Villoro, Luis. El conocimiento, Enciclopedia iberoamericana de filosofía, № 20, Trotta y CSIC, Madrid 1999. 